Research Paper

\title{
Differential Response of Non-cancerous and Malignant Breast Cancer Cells to Conditioned Medium of Adipose tissue-derived Stromal Cells (ASCs)
}

\author{
Yi-Chia Wu ${ }^{1,2,3,4^{*}}$, Wei-Ting Wang ${ }^{*}$, Li-Ju Huang5, Ruo-You Cheng1, Yur-Ren Kuo ${ }^{1,3,6}$, Ming-Feng Hou ${ }^{3,6}$, \\ Chung-Sheng Lai1 13,6 , and John $\mathrm{Yu}^{7,8}{ }^{\boxplus}$
}

1. Division of Plastic Surgery, Department of Surgery, Kaohsiung Medical University Hospital, Kaohsiung, Taiwan

2. Department of Plastic Surgery, Kaohsiung Municipal Ta-Tung Hospital, Kaohsiung, Taiwan

3. Graduate Institute of Medicine, Kaohsiung Medical University, Kaohsiung, Taiwan

4. PhD. Programme in Translational Medicine, Kaohsiung Medical University, Kaohsiung, and Academia Sinica, Taipei, Taiwan

5. Center of Teaching and Research, Kaohsiung Municipal Ta-Tung Hospital, Kaohsiung, Taiwan

6. Faculty of Medicine, College of Medicine, Kaohsiung Medical University, Kaohsiung, Taiwan

7. Institute of Stem Cell and Translational Cancer Research, Chang Gung Memorial Hospital at Linko, Taoyuan, Taiwan

8. Institute of Cellular and Organismic Biology, Academia Sinica, Taipei, Taiwan

* These authors contributed equally to this work.

$\square$ Corresponding author: John Yu, MD., PhD., No.5, Fuxing St., Guishan Dist., Taoyuan City 333, Taiwan. Tel.: 886-3-328-1200\#5218; Fax: 886-3-328-1200\#5214; E-mail: johnyu@gate.sinica.edu.tw

(C) Ivyspring International Publisher. This is an open access article distributed under the terms of the Creative Commons Attribution (CC BY-NC) license (https://creativecommons.org/licenses/by-nc/4.0/). See http://ivyspring.com/terms for full terms and conditions.

Received: 2018.05.07; Accepted: 2019.04.03; Published: 2019.06.02

\begin{abstract}
Background: The application of adipose tissue-derived stromal cells (ASCs) in regenerative medicine has become a growing trend due to its abundance and differentiation potentials. However, several breast cancer studies indicated that ASCs promote tumor progression, therefore, the use of ASCs for reconstruction after oncological surgery poses potential risks. In this study, we aimed to examine whether cancerous or non-cancerous breast cells will exhibit different responses to ASC-derived CM.

Methods: ASCs were isolated from residuals of subcutaneous adipose tissue obtained from patients undergoing surgery. Cancerous MCF-7, MDA-MB231, and MDA-MB468 cell lines and one non-cancerous M10/H184B5F5 cell line were cultured with variant concentrations of ASC-derived conditioned medium (CM) for analysis.

Results: ASC-derived CM significantly reduced cell viability by triggering apoptosis in MCF-7, MDA-MB231, and MDA-MB468 cell lines. ATM-Chk2-dependent DNA damage response was activated early in cancer cells when exposed to ASC-derived CM. By contrast, prompted cell proliferation instead of cell death was detected in M10/H184B5F5 cells under the treatment of lower CM concentration. Even when exposed to the highest concentration of $\mathrm{CM}$, only cell cycle arrest accompanied by a weak DNA damage response were detected in M10/H184B5F5 cells, no cell deaths were observed.

Conclusions: Overall, this study demonstrated that cancerous and non-cancerous breast cells respond differently to ASC-derived CM. ASC-derived CM triggered significant cell death in breast cancer cell lines, however non-cancerous breast cells exhibited dissimilar response to ASC-derived CM.
\end{abstract}

Key words: adipose tissue-derived stromal cell; ASCs; breast cancer cell line; apoptosis

\section{Introduction}

In recent medical practices, the use of bone marrow-derived mesenchymal stem cells (MSCs), particularly adipose tissue-derived stromal cells (ASCs), has attracted growing attentions because of its availability and differentiation potential of ASCs. ASCs has the ability to differentiate into various lineages, including adipogenic, chondrogenic, osteogenic, and hepatocytic cell types as MSCs [1]. Paracrine secretion of cytokines and chemokines as well as growth factors from ASCs have also been reported to exhibit anti-apoptotic, anti-inflammatory, immunomodulatory, and anti-scarring effects [2-3]. 
These specific characteristics make ASCs a promising candidate in a broad array of medical applications. However, the effects of MSCs and ASCs on various types of cancer remain controversial [4]. Several experimental studies support the propensity of MSCs and ASCs to promote growth, progression, and metastasis in various cancer types, including breast cancer, osteosarcoma, and brain tumor [2, 5-6]. Few literature support progressions in other types of cancer [7-9].

In breast surgery, compared with breast prostheses, reconstruction with autologous tissue, such as latissimus dorsi or rectus abdominis, and fat grafting are commonly used, with a benefit of a more natural appearance following oncological mastectomy. However, fat necrosis or apoptosis can lead to transplanted graft resorption and loss of volume, which can ultimately produce an unsatisfactory long-term result [10,11]. Supplementation with ASCs isolated from adipose tissue has been proposed to improve the engraftment and overcome this challenge $[10,12]$.

Given its great potential, a thorough understanding on the influence of ASCs on tumor progression, particularly in breast cancer, may help in exploring the safety of using ASCs in future regenerative medicine and cell therapy. In this study, we showed that non-tumor cells and cancerous breast epithelial cells exhibited different responses to ASC-derived conditioned medium (CM). ASC-derived CM yielded typical DNA damage responses and eventually apoptosis in malignant tumor cells, whereas non-cancerous M10/H184B5F5 cells showed enhanced proliferation in response to lower concentration of ASC-derived CM. Even under the highest concentration of ASC-derived $\mathrm{CM}$, no deaths were detected in M10/H184B5F5 cells.

\section{Material and Methods}

\section{Human ASCs isolation and culture}

ASC isolation in this study was approved by the Institutional Review Board of Kaohsiung Medical University Hospital (KMUH-IRB-F(I)-20170077). Informed consent was obtained from individuals prior to the surgery. Residual subcutaneous adipose tissue was obtained from patients undergoing liposuction surgery. ASCs were further isolated from residuals of adipose tissue and cultured with Defined Keratinocyte serum-free medium (Gibco 10744-019, MA, USA)-based complete K-Medium by following a previously described protocol [13]. ASC-derived CM was collected from culture medium no later than five passages of ASCs. Surface markers of isolated ASCs were examined using an LSR II flow cytometer (BD
Biosciences, NJ, USA) with FlowJo software (FlowJo LLC, OR, USA). The lineage differentiation ability of ASCs was confirmed by adipogenic, osteogenic and chondrogenic induction for 3 weeks followed by Oil Red O (adipogenesis), Alizarin Red S (osteogenesis) and Alcian blue (chondrogenesis) staining (Supplementary Figure S1).

\section{Cell lines}

Four breast cell lines were analyzed: three malignant MCF-7, MDA-MB231, and MDA-MB468 cell lines and one non-cancerous M10/H184B5F5 breast epithelial cell line. While MCF-7 is the most common breast cancer cell line used to assess cancer progression with a relatively low invasiveness, MDA-MB231 is a triple negative (estrogen receptor, progesterone receptor, and HER2 negative) breast cancer cell line with a more malignant potency [2]. In addition, another breast cancer cell lines, MDA-MB468 and the non-cancerous M10/H184B5F5 breast epithelial cells in this study were used for comparisons. MCF-7, MDA-MB231, and M10/H184B5F5 were purchased from Bioresource Collection and Research Center, Taiwan; MDA-MB468 was purchased from American Type Culture Collection, USA. All cell lines in this study were maintained with Dulbecco's modified Eagle's medium (DMEM) supplemented with 10\% FBS (Gibco 26140-079) and 1\% antibiotic-antimycotic, 100× (Gibco 15240-062). Complete DMEM was used as the control medium (ctrl) relative to ASC-derived CM.

\section{Cell cycle analysis}

Treated samples were prepared as previously described [14], and the cell cycle distribution was analyzed using an LSR II flow cytometer with FlowJo software.

\section{Immunofluorescence staining}

Cells were grown on cover slips inside 6-well plates. After treatment, cell-grown slips were fixed with $3 \%$ buffered paraformaldehyde for immunostaining which were prepared as described previously [14]. Fluorescence images were detected and captured using a Zeiss AxioPlan 2 system (Carl Zeiss, Oberkochen, Germany).

\section{Cell viability assay}

Cell viability after treatment was determined using an MTT assay. Treated cells were washed with PBS and incubated with MTT $(10 \mu \mathrm{g} / \mathrm{mL})$ in freshly refed medium under $37^{\circ} \mathrm{C}, 5 \% \mathrm{CO}_{2}$ for 2 hours. Formazan crystal produced by living cells were dissolved in dimethyl sulfoxide and quantified by measuring absorbance at a wavelength of OD570. 


\section{Western blot}

Whole-cell protein lysates of treated cells were extracted and fractionated using sodium dodecyl sulfate-polyacrylamide gel electrophoresis and blotted onto nitrocellulose as previously described [14]. Protein expression was determined using the Western Lightning Plus-ECL detection system (NEL104001EA, PerkinElmer, MA, USA). Primary antibodies used: $\gamma$-H2AX (ab2893, abcam), activated caspase3 (ab32042, abcam), CHK2 pThr 68 (2661, Cell Signaling), CHK2 (3440, Cell Signaling), ATM pSer 1981 (5883, Cell Signaling) and GAPDH (MAB374, Millipore). Secondary antibodies used: anti-rabbit IgG, HRP-linked antibody (7074, Cell Signaling) and anti-mouse IgG, HRP-linked antibody (7076, Cell Signaling).

\section{Statistical analysis}

SPSS 10.0 (one way analysis of variance followed by post hoc analysis with Bonferroni correction; SPSS Inc., Chicago, IL, USA) was used for statistical analysis. Values are presented as means \pm s.d. from three independent experiments. $\mathrm{P}<0.05$ was considered statistically significant between categories.

\section{Results}

\section{ASC-derived CM reduced cell proliferation/viability in MCF-7, MDA-MB231, and MDA-MB468 tumor cells}

To determine the influence caused by culture medium alteration, we firstly examined the apoptotic state of both the most malignant MDA-MB231 and the non-cancerous M10/H184B5F5 cells under either DMEM or fresh K-Medium (FM) which is the basal medium of ASC-derived CM. $5 \times 10^{5}$ MDA-MB231 or M10/H184B5F5 cells were cultured with DMEM or FM for 72 hours followed by flow cytometry analysis. Both floating and attached cells were collected for propidium iodide (PI) staining, commonly used for detecting DNA content in cell cycle analysis. In this study we used PI staining with flow cytometry to examine the population of cell death (sub-G1 population) because small DNA fragments could be observed during the late stage of apoptosis. As shown in the presented results, these two different basal medium triggered no significant difference of sub-G1 populations even in malignant MDA-MB231 (supplementary Figure S2). Furthermore, MCF-7, MDA-MB231, and MDA-MB468 cell lines were

A

MCF-7
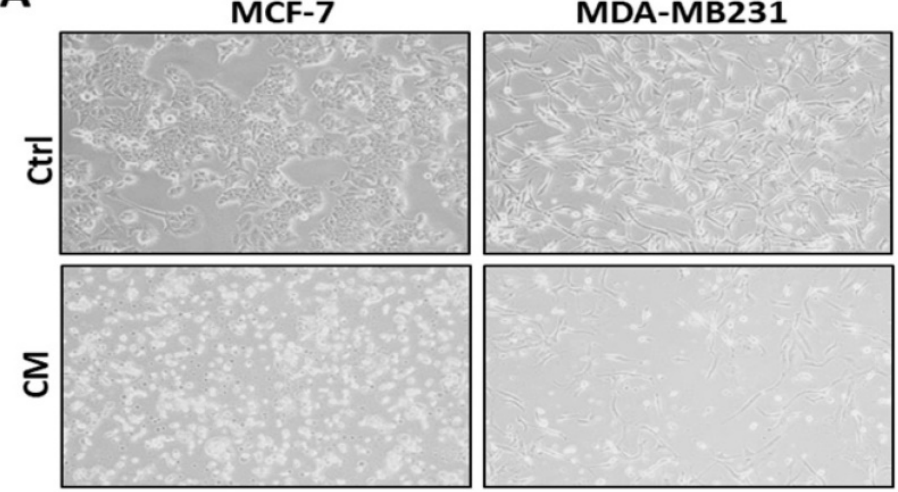

MDA-MB468
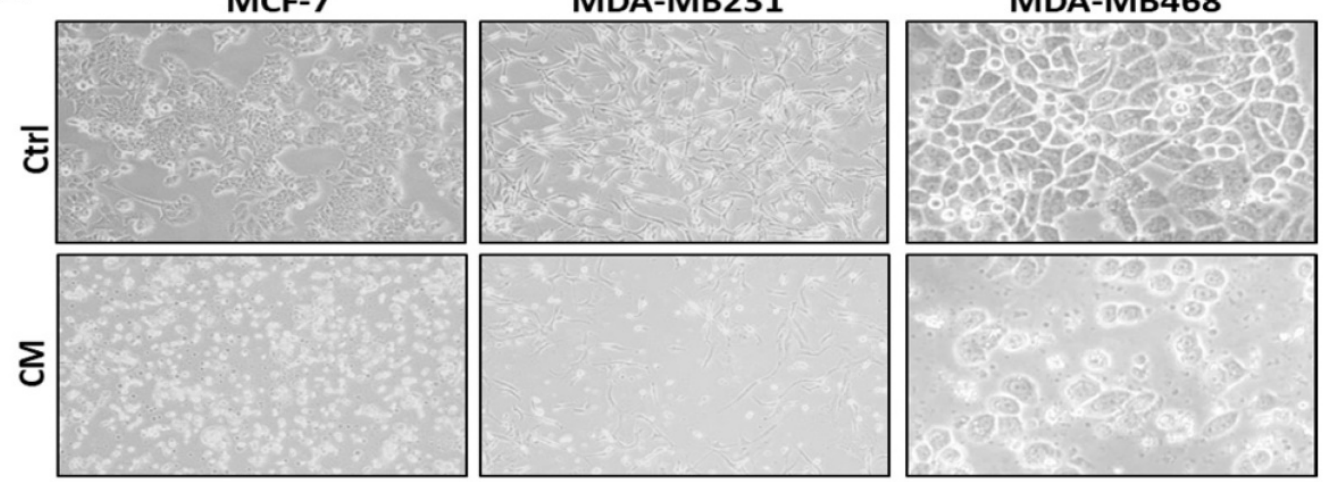

B
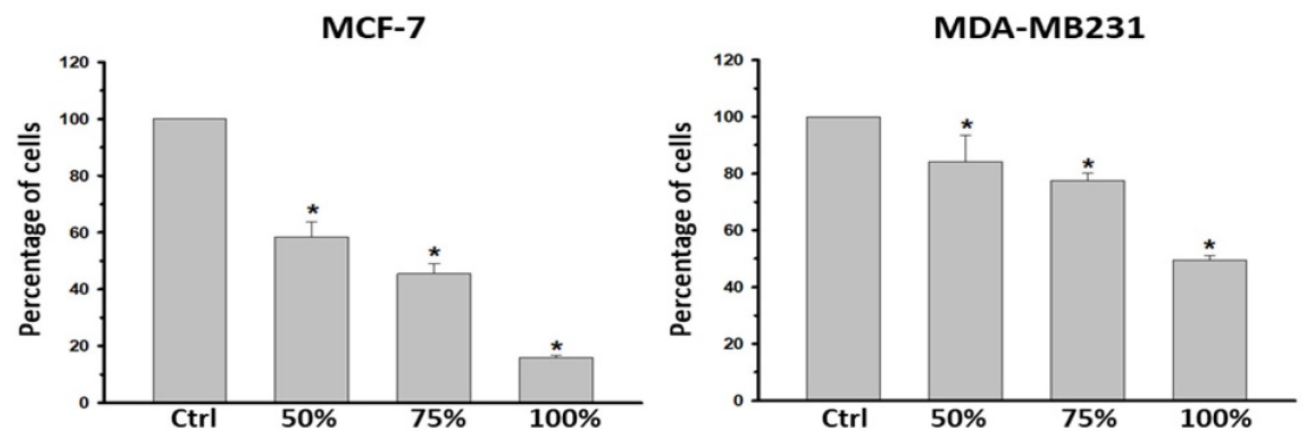

Figure 1. ASC-derived conditioned medium (CM) suppressed cell proliferation and viability in MCF-7, MDA-MB231 and MDA-MB468 breast cancer cells. MCF7, MDA-MB231 and MDA-MB468 cells were cultured with control culture medium or conditioned K-medium (CM) for 72 hours. A) Cell morphology was observed and recorded through microscopy analysis. Magnification: MCF-7 and MDA-MB231: $\times 200 ;$ MDA-MB468: $\times 400$. B) Cell proliferation and viability were examined using the MTT assay. $1 \times 10^{5}$ cells were cultured with control or ASC-derived CM as indicated concentrations for 72 hours. Presented values of bar graphs represent the mean of three independent experiments \pm s.d. $* \mathrm{p}<0.05$ compared with control cells. 
treated with $\mathrm{CM}$ derived from the incubation of $1 \times 10^{6}$ ASCs for $24 \mathrm{~h}$. The CM was filtered with $0.2 \mu \mathrm{m}$ filter to avoid contamination before using for cell culture. All three breast cancer cell lines exhibited a significant reduction in viability when cultured with $\mathrm{CM}$. The morphology of the attached cells became rounder, and an increased number of floating cells was observed, indicating cell death (Figure 1A). The morphological observations were confirmed using an MTT analysis. To avoid any false-positive effect caused due to deficient nutrition in $\mathrm{CM}$, we tested the influence of ASC-derived CM following a dose-dependent manner. ASC-derived CM was mixed with fresh DMEM in proportion for the experiments. As expected, all concentrations (50-100\%) of ASC-derived CM reduced cell viability after $72 \mathrm{~h}$ exposure in both MCF-7 and MDA-MB231 cancer cells (Figure 1B). The similar results were also obtained in MDA-MB468 (supplementary Figure $\mathrm{S} 3 \mathrm{~A})$.

\section{ASC-derived CM triggered cell apoptosis in MCF-7, MDA-MB231, and MDA-MB468 tumor cells}

To further examine the responses of these cells to ASC-derived CM, cells were harvested after treatment and DNA content was analyzed using flow cytometry. In brief, $5 \times 10^{5}$ cells were seeded into 6-well culture dishes overnight to allow attachment, and treated with ASC-derived CM by following a time-course protocol. As shown in Figure 2, the quantification of cell cycle analysis indicated that the sub-G1 population drastically increased and reached a peak after $72 \mathrm{~h}$ of treatment with ASC-derived CM in MCF-7, MDA-MB231 (Figure 2A), and MDA-MB468 (Supplementary Figure S3B) breast cancer cell lines. Consistent with the results obtained from flow cytometry, the expression of cleaved-caspase 3, which is activated during cell apoptosis was also demonstrated (Figure 2B, 2C).

\section{DNA damage appeared in MCF-7 and MDA-MB231 tumor cells when cultured with ASC-derived CM}

We next examined whether cell death triggered by ASC-derived $\mathrm{CM}$ was accompanied by unfavorable DNA damage. We performed immunofluorescence assays for pSer139 histone $\mathrm{H} 2 \mathrm{AX}(\gamma-\mathrm{H} 2 \mathrm{AX}), \gamma-\mathrm{H} 2 \mathrm{AX}$ is recruited when DNA damage occurs, particularly when double-strand breaks occur. It is widely accepted as a marker for DNA damage. MCF-7 and MDA-MB231 cells cultured with ASC-derived $\mathrm{CM}$ for 72 hours exhibited the expression of pan-nuclear $\gamma-\mathrm{H} 2 \mathrm{AX}$ staining relative to cells treated for 72 hours with control culture medium
(Figure 3A). This DNA damage response was further confirmed using a protein immunoblot. In both cells, the protein immunoblot of $\gamma-\mathrm{H} 2 \mathrm{AX}$ was more intense than that of the control group in response to such treatment (Figure 3B, 3C).

\section{No cell death was identified in non-cancerous breast cell lines under ASC-derived CM treatment despite detection of little DNA damage}

Given our evidence that ASC-derived CM led to DNA damage and cell apoptosis in MCF-7 and MDA-MB231 cancer cell lines, we investigated whether non-tumorous cells responded similarly. $1 \times$ $10^{5} \mathrm{M} 10 / \mathrm{H} 184 \mathrm{~B} 5 \mathrm{F5}$ cells were seeded into 6-well culture dishes overnight to allow attachment, and treated with ASC-derived CM as a dose-dependent manner for 72 hours for MTT analysis. Even though ASC-derived CM resulted in a significant decrease of cell population in the cells after 72 hours of exposure, the lower concentration (50 and $75 \%$ ) of ASC-derived $\mathrm{CM}$ dramatically enhanced cell proliferation (Figure 4A). Remarkably, no evidence of cell apoptosis was detected in M10/H184B5F5 cells even exposed to the highest concentration of ASC-derived CM. The cells exhibited only an accumulation of cells with DNA characteristic of the arrested progression in the G1 phase after treatment for the first $24 \mathrm{~h}$. Few cells exhibited sub-G1 DNA content characteristic of apoptotic cells (Figure 4B). Consistent with this finding, no activated- caspase 3 was evident, and only weakly- expressed $\gamma-\mathrm{H} 2 \mathrm{AX}$ was detected in M10/H184B5F5 cells following the 72-h ASC-derived $\mathrm{CM}$ incubation (Figure 4C). Taken together, these data demonstrate entirely distinct effects of ASC-derived $\mathrm{CM}$ on non-cancerous breast epitheliums. Even the highest concentration of ASC-derived CM merely resulted in delayed cell cycle progression in M10/H184B5F5 rather than apoptosis.

\section{Fully- activated ATM-Chk2 signaling cascades in malignant MCF-7 and MDA-MB231, but not in non-cancerous M10/H184B5F5 cells when exposed to ASC-derived CM}

Upon DNA damage, the ataxia telangiectasia mutated (ATM)-Chk2 signal transduction cascade is activated to phosphorylate a number of target proteins to arrest cell cycle progression and trigger DNA repair systems or cell apoptosis to ensure DNA integrity [15-16].

To determine whether this checkpoint system might be related to the induction of DNA damage and cell death by ASC-derived CM, MCF-7, MDA-MB231, and M10/H184B5F5 were cultured with ASC-derived $\mathrm{CM}$ in a time-course manner and then harvested for 
Western blot analysis. The results revealed that MCF-7 and MDA-MB231 cells exhibited typical DNA damage checkpoint activation. Both pSer1981 ATM and pThr68 Chk2 were phosphorylated within 24 hours following $\mathrm{CM}$ exposure. By contrast, this pattern was greatly delayed in M10/H184B5F5 cells, and pThr68 Chk2 activation was only weakly detected after a 72-hours treatment with a slight increase in pSer1981 ATM phosphorylation (Figure 5). Taken together, these data revealed the distinct responses between malignant tumor and non-cancerous cells when exposed to ASC-derived CM.

A
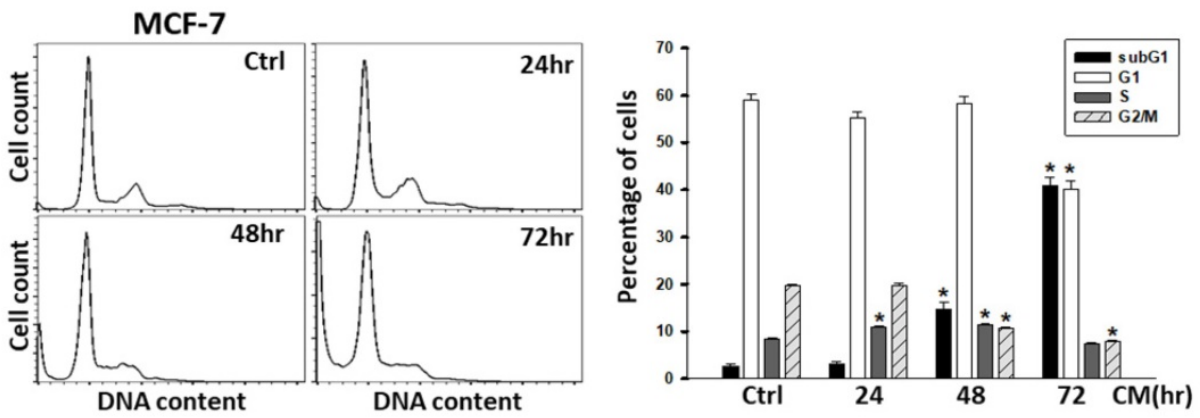

MDA-MB231
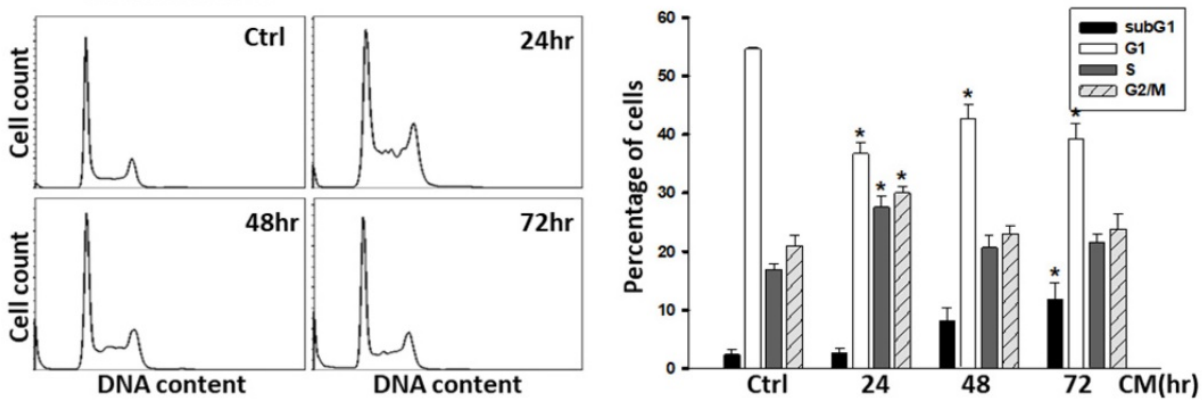

\section{Discussion}

In the practice of plastic surgery, ASCs combined with fat graft have been frequently used in oncological breast reconstruction [17]. However, many in vitro and in vivo studies have pointed out the potential risk of ASCs in promoting breast cancer progression [2,18]. In this regard, questions arise whether the ASCs used in the breast reconstruction may potentially interact with the remaining cancer cells and promote its growth. In our study, ASC-derived CM exhibited inhibitory effects on breast cancer cell lines with increased DNA damage and cell apoptosis.

B

$100 \% \mathrm{CM}$

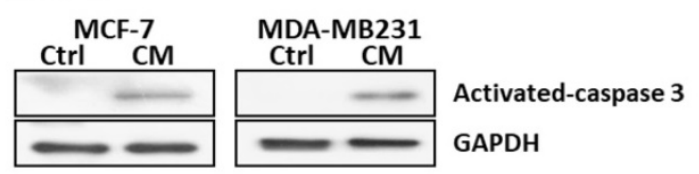

C

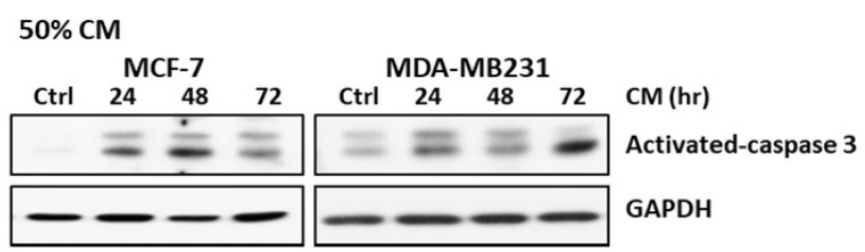

Figure 2. ASC-derived conditioned medium (CM) enhanced apoptosis in MCF-7 and MDA-MB231 breast cancer cells. A) MCF7 and MDA-MB231 cells were cultured with control culture medium or $100 \%$ conditioned K-medium (CM) for indicated time points before harvested, and the cell cycle distribution was analyzed using flow cytometry. In each panel, cell cycle profiles are presented together with bar graphs, indicating the cell distribution in each phase of the cell cycle. Presented values represent the mean of three independent experiments \pm s.d. ${ }^{*}$ p $<0.05$ compared with control. B) Western blot analysis of activated-caspase-3 in MCF-7 or MDA-MB231 cells cultured with $100 \%$ ASC-derived CM for 72 hours. C) Western blot analysis of activated-caspase-3 in MCF-7 or MDA-MB231 cells cultured with $50 \%$ ASC-derived CM as indicated time points. GAPDH levels are presented as loading controls. 
A

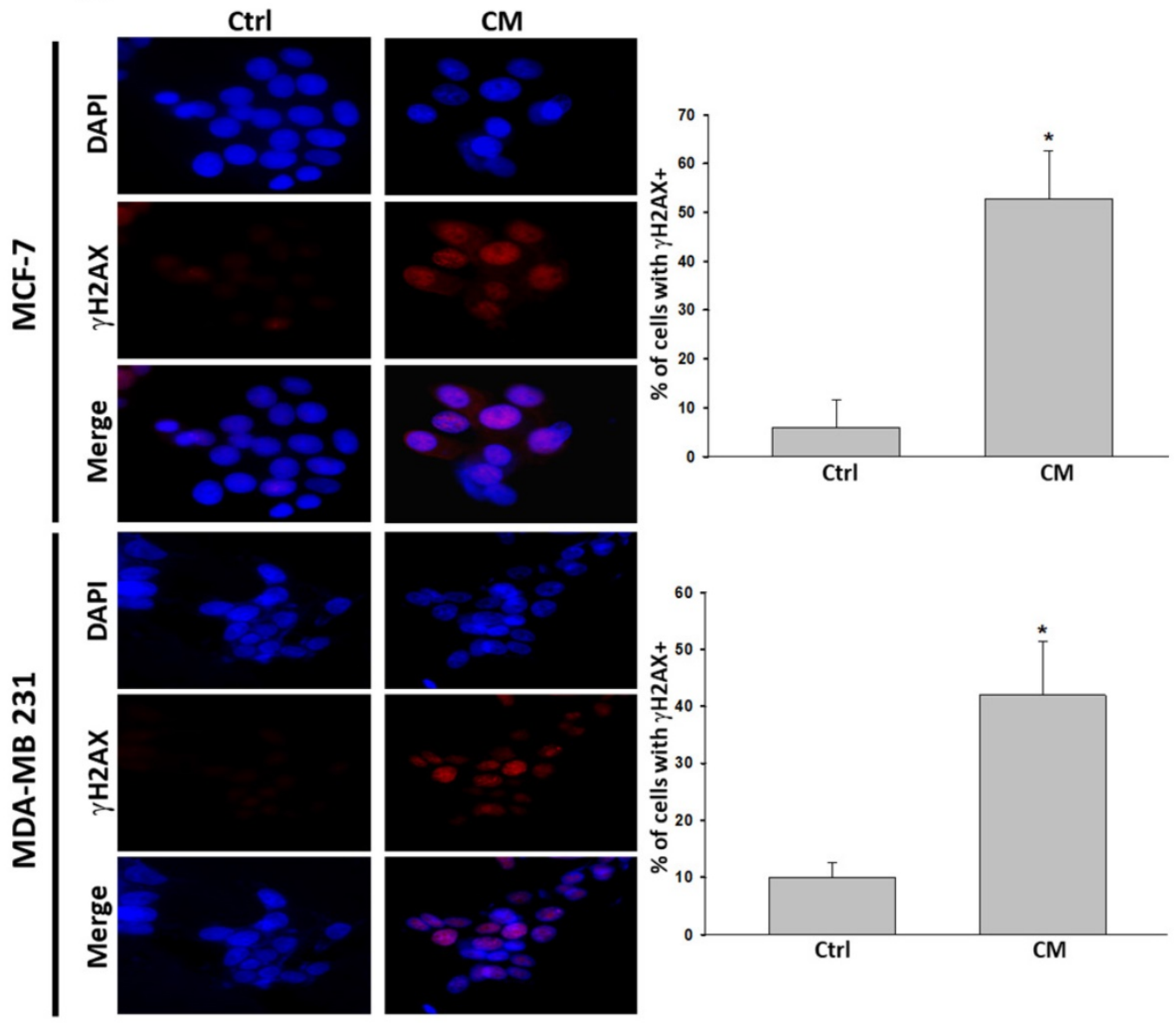

B

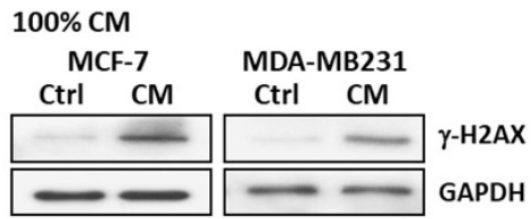

C

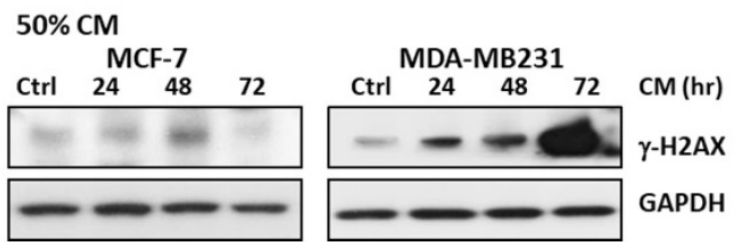

Figure 3. ASC-derived conditioned medium (CM) induced DNA damage in MCF-7 and MDA-MB231 breast cancer cells. A) MCF-7 and MDA-MB231 cells were cultured with $100 \%$ ASC-derived CM for 72 hours before being fixed and stained for DNA content and phosphorylated pSer139 $\mathrm{H} 2 \mathrm{AX}(\gamma-\mathrm{H} 2 \mathrm{AX}$ ). Images from the immunofluorescence analysis of $\gamma-\mathrm{H} 2 \mathrm{AX}$ positive cells in MCF-7 and MDA-MB231 are presented together with bar graphs, indicating the percentage of the mean of three independent experiments \pm s.d. *p $<0.05$ compared with control. B) Western blot analysis of $\mathrm{Y}-\mathrm{H} 2 \mathrm{AX}$ in extracts prepared from MCF-7 and MDA-MB231 cells cultured with $100 \%$ ASC-derived CM for $72 \mathrm{~h}$. C) Western blot analysis of $\mathrm{Y}-\mathrm{H} 2 \mathrm{AX}$ in extracts prepared from MCF-7 and MDA-MB231 cells cultured with $50 \%$ ASC-derived CM for indicated time points. GAPDH levels are presented as loading controls.

The toxic metabolic waste products or the lack of nutrition in the $\mathrm{CM}$ is not the reason for the inhibitory effect observed in this study, since $50 \%$ or $75 \%$ ASC-derived CM also suppressed cell viability on breast cancer cell lines. While different research groups demonstrated various responses in different cancer cell types when interacting with ASC-derived
$\mathrm{CM}$, the discrepancy between these studies may have resulted from dissimilar ASC origins or different culture conditions [2]. In addition to ASCs, mesenchymal stromal cells in other studies also support the inhibitory effects on breast cancer cell lines with either direct co-culture or $\mathrm{CM}$ exposure $[19,20]$, even in highly malignant cell line such as 
MDA-MB231 [21]. Most importantly, no evidence of increased cancer recurrence rate was noted in breast reconstruction with fat grafts which contain ASCs

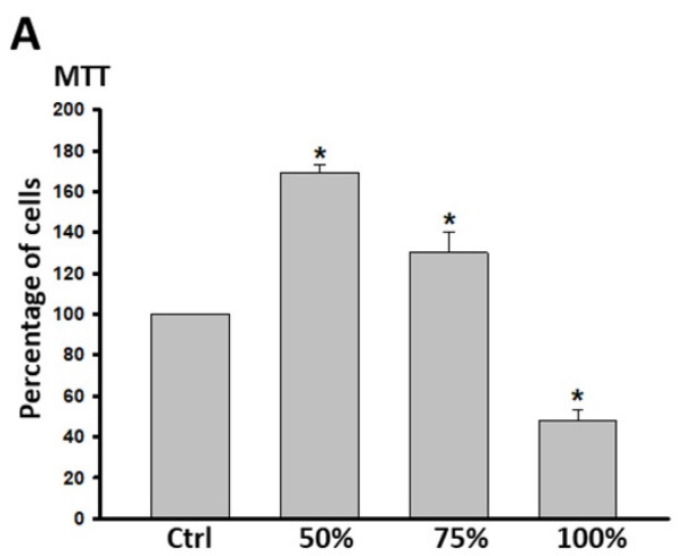

B

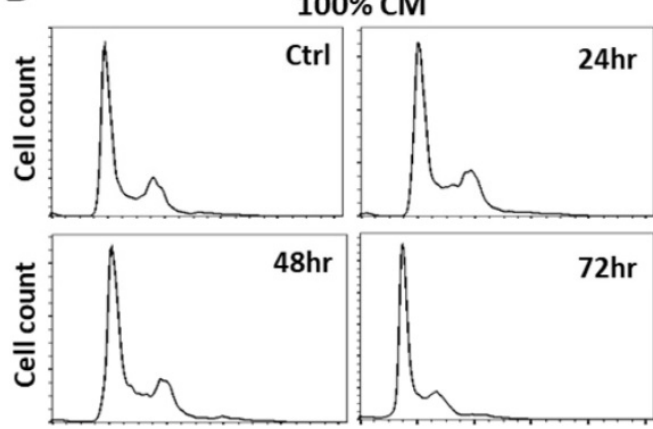

$50 \% \mathrm{CM}$
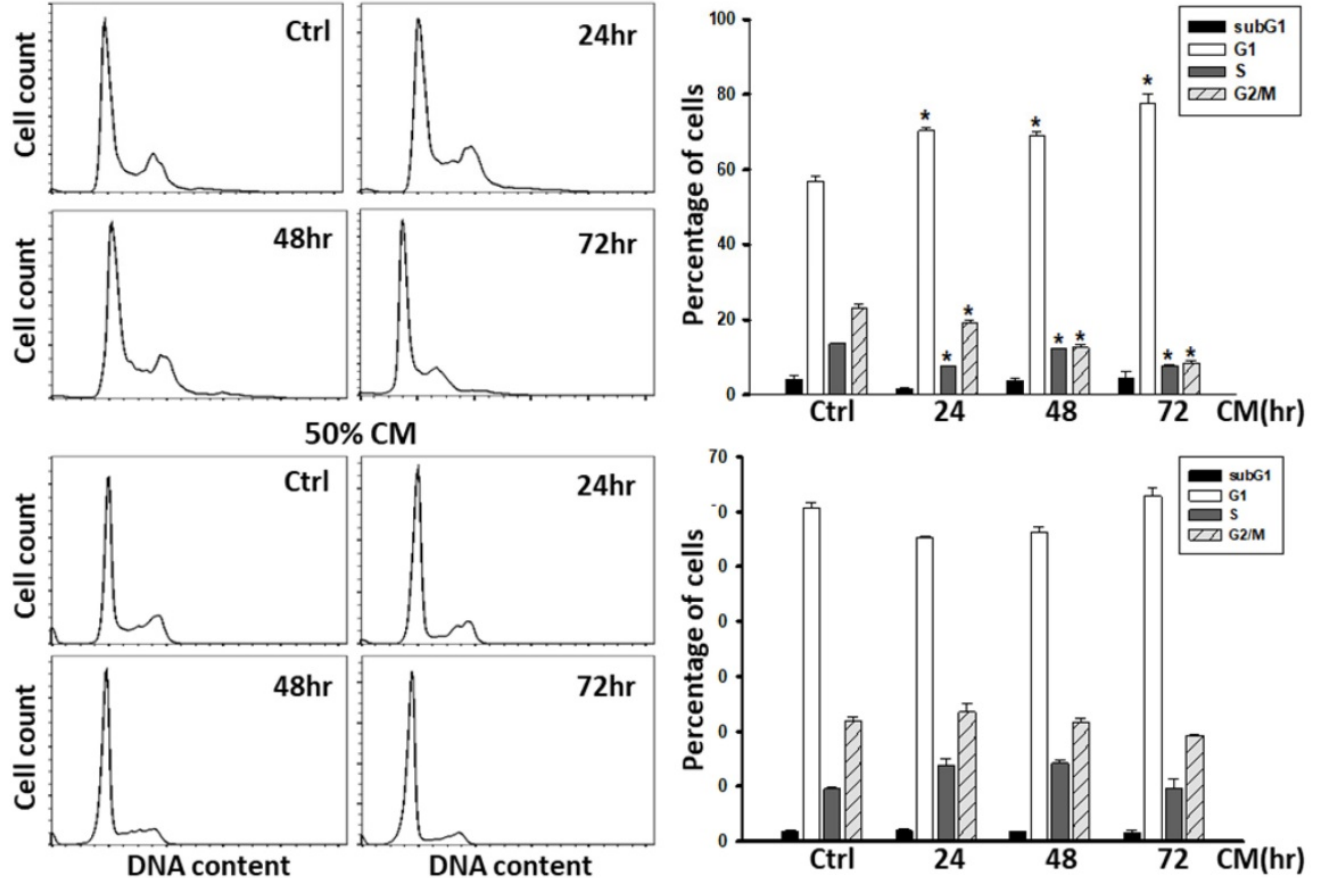

C

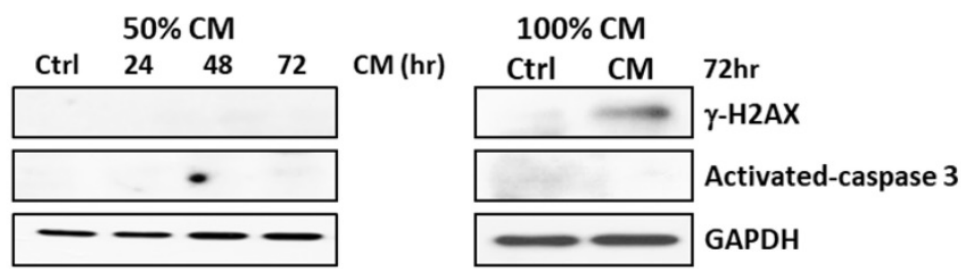

Figure 4. ASC-derived conditioned medium (CM) failed to induce apoptosis in M10/H184B5F5 cells. A) M10/H184B5F5 cells were cultured with control culture medium or conditioned K-medium (CM) as a dose-dependent manner for 72 hours before MTT analysis. Presented values represent the mean of three independent experiments \pm s.d. *p $<0.05$ compared with control. B) M10/H184B5F5 cells were cultured with control culture medium or 100\% conditioned K-medium (CM) for indicated time points before harvest, and the cell cycle distribution was analyzed using flow cytometry. In each panel, cell cycle profiles are presented together with bar graphs, indicating the cell distribution in each phase of the cell cycle. Presented values represent the mean of three independent experiments \pm s.d. C) Western blot analysis of $\gamma$-H2AX and activatedcaspase-3 in M10/H184B5F5 cells cultured with ASC-derived CM for indicated time points. GAPDH levels are presented as loading controls. 


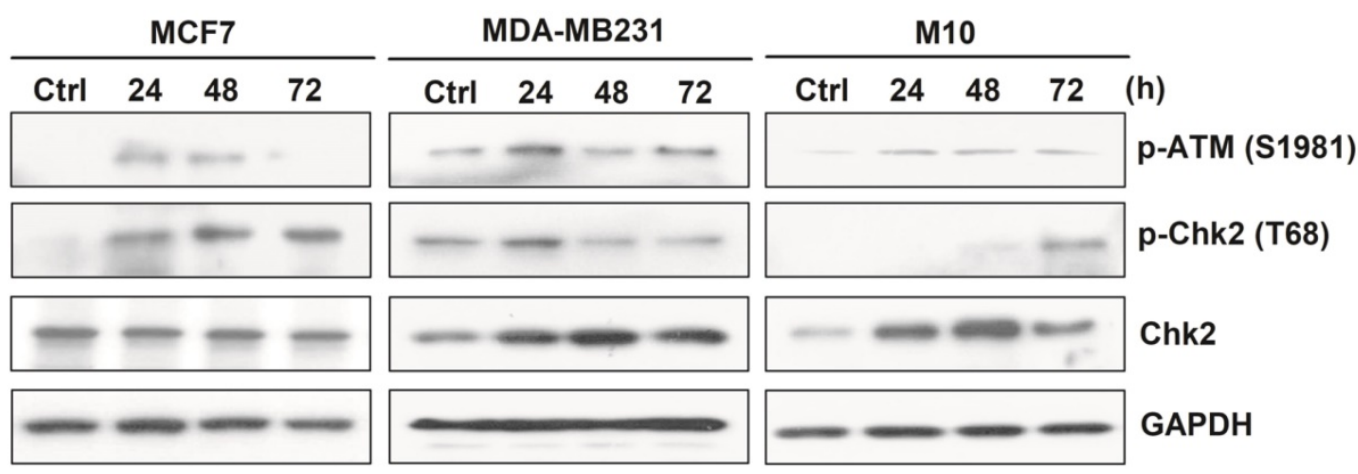

Figure 5. ASC-derived conditioned medium (CM) induced fully activated ATM-Chk2 cascades in MCF-7 and MDA-MB231, but not in M10/H184B5F5 cells. MCF-7, MDA-MB231, and M10/H184B5F5 cells were cultured with ASC-derived CM for up to 72 hours before the harvest and preparation of cell-free extracts. Phosphorylated pSer1981 ATM and pThr68 Chk2 and the total protein level of Chk2 were analyzed using Western blot. GAPDH levels are presented as loading controls.

Notably, our data also revealed that the ATM-Chk2 cascades were activated early by 24 hours in both MCF-7 and MDA-MB231 breast cancer cell lines when exposed to ASC-derived CM. This DNA damage response and the inhibitory effects of ASC-derived CM on tumor cell growth, cell cycle progression, and apoptosis may be resulted from the paracrine effect of ASCs. Some studies showed that the inhibition of cancer cell line maybe related to the increased level of transforming growth factor-beta (TGF- $\beta$ ) $[5,25]$ which is produced and released by ASCs [26]. Furthermore, our results suggested that there may be other undefined mechanisms that protect non-cancerous M10/H184B5F5 cells against stress caused by ASC-derived CM, because these cells did not exhibit fully activated DNA damage signaling and the treatment produced only minimal cell death. In summary, our study evidently showed that ASC-derived CM leads to DNA damage, signaling activation of DNA damage, and eventually cell apoptosis in breast cancer cell lines. By contrast, no cell apoptosis was observed in the non-cancerous breast cell lines when exposed to identical conditions. This study provides additional information on the ongoing debate on the potential risk of using ASCs in breast reconstruction following oncological surgery, however, additional data and further detailed analysis such as the effect of cell-cell contact in ASCs and breast cancer cells are warranted.

\section{Supplementary Material}

Supplementary figures.

http://www.medsci.org/v16p0893s1.pdf

\section{Acknowledgments}

We would like to express our gratitude to the Center for Research Resources and Development (CCRD) of Kaohsiung Medical University for the technical assistance. This study was partially funded by grants from CGMH at Linko of Taiwan to Dr. John
$\mathrm{Yu}$ (OMRPG3C0041 to OMRPG3C0044); Ministry of Science and Technology, Taiwan to Dr. Yi-Chia Wu (MOST 103-2628-B-037-002-MY3); Kaohsiung Municipal Ta-Tung Hospital to Dr. Li-Ju Huang (kmtth104-046) and Dr. Yi-Chia Wu (kmtth-105-011; kmtth104-011); grants from Kaohsiung Medical University Hospital to Dr. Yi-Chia Wu (kmuh98-8G42 and kmuh99-9M54); and grants from Academia Sinica, Taiwan to Dr. Yi-Chia Wu (AS-TM-108-02-01). This manuscript was edited by Wallace Academic Editing. We also thank Dr. Tzu-Yu Lin for constructive criticism of the manuscript.

\section{Competing Interests}

The authors have declared that no competing interest exists.

\section{References}

1. Zuk PA, Zhu M, Mizuno $\mathrm{H}$, et al. Multilineage cells from human adipose tissue: Implications for cell-based therapies. Tissue Eng. 2001; 7: 211-228.

2. Schweizer R, Tsuji W, Gorantla VS, et al. The role of adipose-derived stem cells in breast cancer progression and metastasis. Stem Cells Int. 2015; 2015: 120949.

3. Zuk PA. The adipose-derived stem cell: Looking back and looking ahead. Mol Biol Cell. 2010; 21: 1783-1787.

4. Klopp AH, Gupta A, Spaeth E, et al. Concise review: Dissecting a discrepancy in the literature: do mesenchymal stem cells support or suppress tumor growth? Stem Cells. 2011; 29: 11-19.

5. Xu WT, Bian ZY, Fan QM, et al. Human mesenchymal stem cells (hMSCs) target osteosarcoma and promote its growth and pulmonary metastasis. Cancer Lett. 2009; 281: 32-41.

6. Behnan J, Isakson $P$, Joel $\mathrm{M}$, et al. Recruited brain tumor-derived mesenchymal stem cells contribute to brain tumor progression. Stem Cells. 2014; 32: 1110-1123.

7. Cousin B, Ravet E, Poglio S, et al. Adult stromal cells derived from human adipose tissue provoke pancreatic cancer cell death both in vitro and in vivo. PLoS One. 2009; 4: e6278.

8. Takahara K, Ii M, Inamoto T, et al. Adipose-derived stromal cells inhibit prostate cancer cell proliferation inducing apoptosis. Biochem Biophys Res Commun. 2014; 446: 1102-1107.

9. $\mathrm{Yu} \mathrm{X}, \mathrm{Su} \mathrm{B}, \mathrm{Ge} \mathrm{P}$, et al. Human adipose derived stem cells induced cell apoptosis and s phase arrest in bladder tumor. Stem Cells Int. 2015; 2015: 619290.

10. Kolle SF, Fischer-Nielsen A, Mathiasen AB, et al. Enrichment of autologous fat grafts with ex-vivo expanded adipose tissue-derived stem cells for graft survival: A randomised placebo-controlled trial. Lancet. 2013; 382: 1113-1120.

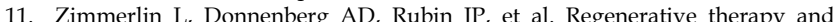
cancer: In vitro and in vivo studies of the interaction between adipose-derived stem cells and breast cancer cells from clinical isolates. Tissue Eng Part A. 2011; 17: 93-106.

12. Yoshimura K, Sato K, Aoi N, et al. Cell-assisted lipotransfer for cosmetic breast augmentation: Supportive use of adipose-derived stem/stromal cells. Aesthet Plast Surg. 2008; 32: 48-55. 
13. Huang SH, Lin YN, Lee SS, et al. New adipose tissue formation by human adipose-derived stem cells with hyaluronic acid gel in immunodeficient mice. Int J Med Sci. 2015; 12: 154-162.

14. Wang WT, Catto JW, Meuth M. Differential response of normal and malignant urothelial cells to CHK1 and ATM inhibitors. Oncogene. 2015; 34: 2887-2896.

15. Stracker TH, Usui T, Petrini JH. Taking the time to make important decisions: The checkpoint effector kinases chk1 and chk2 and the DNA damage response. DNA Repair (Amst). 2009; 8: 1047-1054.

16. Bartek J, Lukas J. Chk1 and chk2 kinases in checkpoint control and cancer. Cancer Cell. 2003; 3: 421-429.

17. Arshad Z, Karmen L, Choudhary R, et al. Cell assisted lipotransfer in breast augmentation and reconstruction: A systematic review of safety, efficacy, use of patient reported outcomes and study quality. J Plast Reconstr Aesthetic Surg Open. 2016; 10: 5-20.

18. Petit JY, Botteri E, Lohsiriwat V, et al. Locoregional recurrence risk after lipofilling in breast cancer patients. Ann Oncol. 2012; 23: 582-588.

19. Kucerova L, Skolekova S, Matuskova M, et al. Altered features and increased chemosensitivity of human breast cancer cells mediated by adipose tissue-derived mesenchymal stromal cells. BMC Cancer. 2013; 13: 535.

20. Qiao L, Xu ZL, Zhao TJ, et al. Dkk-1 secreted by mesenchymal stem cells inhibits growth of breast cancer cells via depression of wnt signalling. Cancer Lett. 2008; 269: 67-77.

21. Sun B, Yu KR, Bhandari DR, et al. Human umbilical cord blood mesenchymal stem cell-derived extracellular matrix prohibits metastatic cancer cell mda-mb-231 proliferation. Cancer Lett. 2010; 296: 178-185.

22. Kronowitz SJ, Mandujano CC, Liu J, et al. Lipofilling of the Breast Does Not Increase the Risk of Recurrence of Breast Cancer: A Matched Controlled Study. Plast Reconstr Surg. 2016; 137: 385-393.

23. Petit JY, Rietjens M, Botteri E, et al. Evaluation of fat grafting safety in patients with intraepithelial neoplasia: a matched-cohort study. Ann Oncol Off J Eur Soc Med Oncol. 2013; 24: 1479-1484.

24. Charvet HJ, Orbay H, Wong MS, et al. The Oncologic Safety of Breast Fat Grafting and Contradictions Between Basic Science and Clinical Studies: A Systematic Review of the Recent Literature. Ann Plast Surg. 2015; 75: 471-479.

25. Hubackova S, Krejcikova K, Bartek J, et al. IL1- and TGF $\beta$-nox4 signaling, oxidative stress and DNA damage response are shared features of replicative, oncogene-induced, and drug-induced paracrine 'bystander senescence'. Aging (Albany NY). 2012; 4: 932-951.

26. Casiraghi F, Remuzzi G, Abbate M, et al. Multipotent mesenchymal stromal cell therapy and risk of malignancies. Stem Cell Rev. 2013; 9: 65-79. 